\title{
Satisfacción Vital en Adolescentes: Relación con la Autoeficacia y el Apoyo Social Percibido.
}

\section{Life Satisfaction in Adolescents: Relationship with Self-efficacy and Perceived Social Support}

\author{
María Paulina Chavarría \\ Enrique Barra \\ Universidad de Concepción, Chile
}

(Rec: 21 diciembre 2012 / Acept: 20 diciembre 2013)

\begin{abstract}
Resumen
Este estudio tuvo como objetivo examinar la relación de las variables autoeficacia y apoyo social percibido con la satisfacción vital en adolescentes. La muestra estuvo conformada por 358 adolescentes de ambos sexos con edades comprendidas entre los 14 y 19 años. Los resultados mostraron altos niveles de autoeficacia y apoyo social percibido, así como un nivel relativamente alto de satisfacción vital. La satisfacción vital mostró relaciones significativas tanto con la autoeficacia como con el apoyo social, siendo algo mayor la relación con la autoeficacia. Se proponen posibles explicaciones para los resultados encontrados. Palabras clave: satisfacción vital, autoeficacia, apoyo social percibido, adolescentes.
\end{abstract}

\begin{abstract}
This study examined the relationship of life satisfaction with self-efficacy and perceived social support in 358 male and female adolescents from Concepcion (Chile), 15 to 19 years old. Results showed high levels of self-efficacy, perceived social support and life satisfaction in this sample. Both self-efficacy and social support had significant positive relationships with life satisfaction, with self-efficacy having more relationship with life satisfaction than perceived social support. Diverse explanatory factors for obtained results are proposed. Key words: life satisfaction, self esteem, social support, adolescents
\end{abstract}

Correspondencia: María Paulina Chavarría Rocha. Departamento de Psicología, Universidad de Concepción, Casilla 160-C, Concepción, Chile. E-mail: mpaulina.chavarria@gmail.com

Investigación financiada por CONICYT. CONICYT-PCHA/Magíster Nacional/2011-22110152 


\section{Introducción}

Tradicionalmente se ha considerado la adolescencia como un período de la vida especialmente problemático y conflictivo (Oliva, Ríos, Antolín, Parra, Hernando y Pertegal, 2010). Sin embargo, la conceptualización actual de la adolescencia parte de una visión más optimista, considerando que los adolescentes no son problemas a ser resueltos, sino valiosos recursos en proceso de desarrollo.

Este enfoque de desarrollo positivo pone énfasis en las necesidades del desarrollo y en las habilidades y actitudes que contribuyen a que los adolescentes se conviertan en personas adultas responsables y capaces (Oliva et al., 2010). El concepto de desarrollo positivo asume que todos los adolescentes tienen fortalezas y que éstas deben identificarse, edificarse y utilizarse por medio de intervenciones efectivas, ya que las fortalezas personales no sólo favorecen la adopción de conductas positivas, sino que alejan a las personas de conductas perjudiciales. Por lo tanto, resulta necesario incluir conceptos aportados por la Psicología Positiva, como felicidad, bienestar subjetivo y satisfacción con la vida, en el estudio de la adolescencia (Giménez, Vasquez y Hervás, 2010; Luna, Laca y Mejía, 2011).

La satisfacción con la vida corresponde al componente cognitivo del constructo de bienestar subjetivo, y generalmente se define como la evaluación general que una persona hace de su propia vida (Diener, Scollon y Lucas, 2009; Vera-Villarroel, Urzúa, Pavez, Celis-Atenas y Silva, 2012). Sin embargo, también es posible concebir y evaluar la satisfacción vital en base a sus distintas dimensiones o ámbitos, tal como lo hace el modelo multidimensional de la satisfacción vital de niños y adolescentes que incluye los dominios familiar, escolar, amigos, sí mismo y entorno (Antaramian, y Huebner, 2009; Huebner, 2001).

Proctor, Linley y Maltby (2009) plantean que es muy importante analizar los factores relacionados a la satisfacción con la vida en los jóvenes, ya que la revisión de investigaciones respecto a este tema muestra que la percepción de satisfacción con la vida en los jóvenes tiene implicaciones importantes para su funcionamiento psicológico, social y educacional.

Algunos factores psicológicos que han sido relacionados con el bienestar subjetivo y la satisfacción vital son la autoeficacia (Reina, Oliva y Parra, 2010; Yusoff, 2012) y el apoyo social (Karademas, 2006; Moreno y Hidalgo, 2010), sin embargo en nuestro medio existe escasez de estudios al respecto, especialmente en población adolescente. Por lo tanto, en un intento de comprender y obtener mayor información respecto al bienestar subjetivo en los adolescentes, este estudio estuvo orientado a examinar la relación de la autoeficacia y el apoyo social percibido con la satisfacción con la vida en esta etapa, desde un modelo multidimensional.

Diversos estudios reportan asociaciones significativas de la autoeficacia con la satisfacción vital, entre ellos el de O'Sullivan (2011) en estudiantes universitarios estadounidenses y el de Yusoff (2012) en universitarios extranjeros en Tailandia. Respecto a estudios con adolecentes, Reina et al. (2010) encontraron que la satisfacción vital de adolecentes españoles entre 12 y 17 años se relacionaba significativamente con la autoeficacia, aunque presentaba una mayor relación con la autoestima.

Aunque en nuestro medio no existen investigaciones que relacionen específicamente autoeficacia con satisfacción vital en adolescentes, algunos estudios han examinado la relación de la autoeficacia con otras variables en adolescentes. Así, Olivari y Barra (2005) informaron que entre las mujeres adolescentes existía una relación inversa entre autoeficacia general y conducta de fumar y que los adolescentes no fumadores presentaban mayor autoeficacia que los fumadores. Por su parte, Avendaño y Barra (2008) encontraron que el nivel de autoeficacia general se relacionaba significativamente con la calidad de vida en adolescentes con enfermedades crónicas. Estas evidencias indican que la autoeficacia sería una de las características personales que promueven el bienestar de las personas, aun en condiciones de adversidad.

El apoyo social percibido se refiere a la valoración subjetiva de la calidad del apoyo social existente, ya sea de tipo instrumental (conductas dirigidas a solucionar un problema), informativo (información útil para afrontar un problema) o emocional (muestras de empatía, amor y confianza (Vega y González, 2009). El apoyo social refuerza la autoestima y favorece una percepción más positiva del ambiente, por lo cual tendría efectos positivos en el bienestar y en la salud al aumentar la resistencia al estrés (Barra, 2004).

En su revisión de la importancia del apoyo social en la adolescencia, Orcasita y Uribe (2010) concluyen que el apoyo social se constituye como factor de protección en el bienestar del adolescente, ya que brinda apoyo emocional, material e informacional, los cuales generan recursos valiosos para enfrentar las situaciones de riesgo que se pueden presentar en esta etapa del ciclo vital.

Diversos estudios en adultos han encontrado una relación positiva entre apoyo social y satisfacción con la vida, como el de Karademas (2006) con adultos griegos, el de Moreno e Hidalgo (2010) con inmigrantes en España, el de Marrero y Carballeira (2010) con adultos españoles y el de Mella, 
González, D’appolonio, Maldonado, Fuenzalida y Díaz, (2004) con adultos mayores chilenos.

Sin embargo, existe menos evidencia acerca de cuál es la relación específica entre el apoyo social percibido y la satisfacción vital de los adolescentes. En un estudio con adolescentes en nuestro medio se encontró que el apoyo social percibido no se relacionaba significativamente con variables asociadas al bienestar individual, como problemas de salud, estrés percibido y ánimo depresivo (Barra, Cerna, Kramm y Veliz, 2006). Mientras que en otro estudio acerca de la calidad de vida de adolescentes con enfermedades crónicas, se encontró que la calidad de vida presentaba una relación muy significativa con la autoeficacia y una asociación mucho menor con el apoyo social percibido (Avendaño y Barra, 2008).

Teniendo en consideración la escasez de investigaciones en nuestro medio que aborden específicamente la satisfacción con la vida en los adolescentes y su relación con otros factores, los objetivos de la presente investigación fueron: 1) describir los niveles de autoeficacia, apoyo social y satisfacción vital de los adolescentes de Colegios Municipales de la comuna de Concepción. 2) examinar las relaciones de la autoeficacia y el apoyo social con la satisfacción vital en dichos adolescentes.

\section{Método}

\section{Participantes}

Para conformar la muestra se seleccionó al azar un $2^{\circ}$ año medio de cada uno de los 13 Colegios Municipales de la comuna de Concepción, con lo cual la muestra final estuvo constituida por 358 adolescentes, 173 hombres y 185 mujeres, con edades entre 14 y 19 años (promedio de 15.7 años).

\section{Instrumentos}

Escala de Autoeficacia General de Bäßler, Schwarzer y Jerusalem (1993, adaptada en Chile por Cid, Orellana y Barriga, 2010): Esta escala consta de 10 ítems con respuestas tipo Likert que van desde Incorrecto (1) a Cierto (4). Mayores puntajes indican mayor nivel de autoeficacia. En cuanto a su confiablidad por consistencia interna, en estudios realizados en Chile se han obtenido coeficientes alfa entre .78 y .91 (Álvarez y Barra, 2010; Avendaño y Barra, 2008; Cid et al., 2010). En este estudio se obtuvo un coeficiente alfa de Cronbach de .80 .

Cuestionario de Apoyo Social Percibido (Gracia, Herrero y Musitu, 2002): Incluye 6 ítems agrupados en
3 dimensiones de apoyo (emocional, consejo y ayuda). El individuo debe indicar las personas relevantes para él o ella y luego debe señalar cuánto apoyo percibe en cada una de esas personas, con cinco opciones de respuesta, desde 1 (Nunca-Casi Nunca) hasta 5 (Siempre-Casi Siempre). Como los participantes pueden incluir diferentes cantidades de personas relevantes, los puntajes son los promedios de las respuestas para cada persona. Mayores puntajes indican mayor nivel de apoyo social percibido. Respecto a sus características psicométricas, los autores informan un coeficiente de consistencia interna alfa de Cronbach igual a .99 y evidencias de validez discriminante en adultos (Gracia et al., 2002). En estudios con adolescentes de nuestro medio se han obtenido coeficientes alfa de Cronbach de .82 (Barra et al., 2006), de .84 (Avendaño y Barra, 2008) y de .98 (Méndez y Barra, 2008). En este estudio se obtuvo un coeficiente alfa de Cronbach de .91.

Escala Multidimensional de Satisfacción con la Vida en Estudiantes (Huebner, 2001): Esta escala permite obtener un perfil de los juicios de los adolescentes respecto a la satisfacción con dominios importantes: familia, colegio, amigos, entorno y uno mismo. Está conformada por 40 ítems con respuestas tipo Likert que van desde Completamente en desacuerdo (1) a Estoy de acuerdo completamente (5). Mayores puntajes indican mayor nivel de satisfacción. Respecto a su confiablidad por consistencia interna, Huebner (2001) informa de coeficientes alfa de Cronbach entre .70 y .90 para las distintas dimensiones. En este estudio se obtuvo un coeficiente alfa de Cronbach de .89 para el instrumento total y coeficientes alfa entre .77 y .87 para las distintas dimensiones.

\section{Procedimiento}

Se realizó primero un estudio piloto con un curso de $2^{\circ}$ año medio no incluido en la muestra final, con el objetivo de verificar la adecuación de los instrumentos para los participantes, no requiriéndose modificaciones. Los instrumentos se aplicaron de manera colectiva durante una clase en cada uno de los 13 cursos seleccionados al azar, previa firma de una carta de consentimiento informado por parte de cada uno de los participantes.

\section{Resultados}

Con el fin de cumplir el primer objetivo de investigación se presentan los estadísticos descriptivos de las variables en la Tabla 1. 
Tabla 1. Estadísticos descriptivos de las distintas variables

\begin{tabular}{lccccc}
\hline & $\begin{array}{c}\text { Puntaje } \\
\text { mínimo }\end{array}$ & $\begin{array}{c}\text { Puntaje } \\
\text { máximo }\end{array}$ & Mediana & Media & DE \\
\hline Autoeficacia & 13 & 40 & 33 & 32.44 & 4.41 \\
Apoyo social global & 2.29 & 5 & 4.33 & 4.22 & 0.57 \\
Apoyo emocional & 2 & 5 & 4.33 & 4.21 & 0.65 \\
Apoyo de consejo & 1.25 & 5 & 4.5 & 4.3 & 0.67 \\
Apoyo instrumental & 1.40 & 5 & 4.29 & 4.15 & 0.70 \\
Satisfacción vital global & 71 & 196 & 157.5 & 154.27 & 19.70 \\
Satisfacción familia & 8 & 35 & 30 & 28.16 & 5.82 \\
Satisfacción colegio & 9 & 40 & 28 & 27.31 & 6.07 \\
Satisfacción amigos & 10 & 45 & 40 & 39.19 & 5.45 \\
Satisfacción entorno & 11 & 45 & 31 & 30.33 & 7.27 \\
Satisfacción uno mismo & 8 & 35 & 30 & 29.27 & 4.80 \\
\hline
\end{tabular}

En la variable de autoeficacia los puntajes pueden variar entre 10 y 40 puntos, por lo cual el promedio observado refleja un nivel alto de autoeficacia en estos adolescentes ( $81 \%$ del máximo posible). En cuanto al apoyo social el puntaje puede variar entre 1 y 5 , por lo cual también se observa un alto nivel en el apoyo global $(84 \%)$ y en cada una de las dimensiones (emocional, consejo e instrumental).

Respecto a la satisfacción vital global, los puntajes pueden variar entre 40 y 200 , por lo cual el promedio observado representa un nivel relativamente alto $(77 \%$ del máximo posible). Los promedios de cada dimensión de satisfacción vital muestran niveles altos en las dimensiones familia ( $80 \%$ ), amigos (87\%) y uno mismo (84\%). En cambio se observan menores niveles de satisfacción en las dimensiones colegio (68\%) y entorno (67\%).

Para abordar el segundo objetivo planteado se calcularon los coeficientes de correlación de Pearson que permiten describir la relación de las variables autoeficacia y apoyo social con la satisfacción vital.

Tabla 2. Correlaciones entre las variables en la muestra total y en cada sexo.

\begin{tabular}{|c|c|c|c|}
\hline & $\begin{array}{c}\text { Satisfacción vital muestra } \\
\text { total }\end{array}$ & Satisfacción vital hombres & Satisfacción vital mujeres \\
\hline Autoeficacia & $0.40 * *$ & $0.46^{* *}$ & $0.38 * *$ \\
\hline Apoyo social global & $0.34 * *$ & $0.34 * *$ & $0.34 * *$ \\
\hline Apoyo emocional & $0.28 * *$ & $0.30 * *$ & $0.26 * *$ \\
\hline Apoyo de consejo & $0.32 * *$ & $0.33^{* *}$ & $0.30 * *$ \\
\hline Apoyo instrumental & $0.27 * *$ & $0.22 * *$ & $0.31 * *$ \\
\hline
\end{tabular}

Nota. $* * \mathrm{p}<.01$

Como se observa en la Tabla 2, tanto la autoeficacia como el apoyo social global y sus dimensiones presentan relaciones significativas con la satisfacción vital en la muestra total y en cada sexo. La autoeficacia muestra una relación algo mayor con la satisfacción vital que el apoyo social, lo cual se aprecia especialmente en los hombres adolescentes. También se puede observar que el apoyo social instrumental es la única variable que muestra mayor relación con la satisfacción vital en las mujeres que en los hombres.

\section{Discusión}

El primer objetivo de este estudio era describir los niveles de autoeficacia, apoyo social percibido y satisfacción vital de los adolescentes estudiados. Los resultados obtenidos revelan niveles altos en las variables de autoeficacia y apoyo social percibido, con promedios grupales sobre el $80 \%$ del máximo posible. En cuanto a la satisfacción vital, se encontró un nivel promedio relativamente alto de satisfacción global 
(77\% del máximo posible) y altos niveles de satisfacción en las dimensiones amigos, uno mismo y familia. En cambio se encontraron menores niveles de satisfacción en las dimensiones colegio y entorno, en comparación con las otras tres dimensiones.

El nivel relativamente alto de satisfacción vital global observado en este grupo de adolescentes es consistente con lo encontrado por Proctor, Linley y Maltby (2009) en su revisión de estudios sobre satisfacción vital en jóvenes. Según estos autores, la mayoría de los estudios muestran que tanto los niños como los adolescentes refieren altos niveles de satisfacción vital. Y el hecho de que en el grupo estudiado la satisfacción con el colegio sea menor que en otras áreas concuerda con lo informado por Galíndez y Casas (2011) en un estudio de la satisfacción vital multidimensional en adolescentes españoles, donde se encontró que los adolescentes estaban muy satisfechos con sus vidas globalmente, y también con sus familias, sus amigos, su entorno y consigo mismos, sin embargo estaban sólo moderadamente satisfechos con la escuela. Esta menor satisfacción con la escuela que con los otros ámbitos podría explicarse porque, en comparación con la familia y los amigos, la escuela representa una institución más formal con normas y relaciones sociales organizadas desde una autoridad jerárquica y donde además existen exigencias establecidas de rendimiento y dedicación a las tareas académicas

El segundo objetivo del presente estudio era examinar las relaciones de la autoeficacia y el apoyo social percibido con la satisfacción vital de los adolescentes. Se encontró que tanto la autoeficacia como el apoyo social global y sus dimensiones presentaban relaciones significativas con la satisfacción vital, siendo la autoeficacia la que presentaba la mayor relación.

La relación observada entre autoeficacia y satisfacción vital concuerda con lo informado por Suldo y Huebner (2006), quienes encontraron que los adolescentes con un alto nivel de satisfacción vital reportaban niveles significativamente mayores de autoeficacia que los adolescentes con un nivel intermedio de satisfacción vital. Y también es consistente con lo informado por O'Sullivan (2011) en estudiantes universitarios estadounidenses y por Reina et al. (2010) en adolescentes españoles. Esta relación se puede explicar porque la evaluación global que un individuo, en este caso adolescente, realiza de sí mismo en términos de su capacidad para actuar efectivamente se encontraría íntimamente relacionada con la valoración que realiza de su vida, de modo que un mayor sentido de autoeficacia se asociaría con mayores índices de satisfacción vital.

La relación entre apoyo social y satisfacción vital encontrada en este estudio también ha sido reportada por otros autores. Suldo y Huebner (2006) encontraron que el apoyo social que los adolescentes percibían en sus padres, profesores, compañeros y amigos se relacionaba significativamente con su nivel de satisfacción vital. Esta relación también ha sido reportada en estudios con adultos, como el de Marrero y Carballeira (2010) con adultos jóvenes y medios españoles y el de Mella et al. (2004) en adultos mayores chilenos. La relación entre apoyo social y satisfacción vital puede explicarse porque el apoyo social permite a la persona lograr una mayor estabilidad, predictibilidad y control, refuerza la autoestima y favorece la construcción de una percepción más positiva de su ambiente (Barra, 2004).

El resultado encontrado en este estudio respecto a que la autoeficacia presenta una mayor relación con la satisfacción vital que el apoyo social sería coincidente con los resultados obtenidos por Yusoff (2012) en universitarios extranjeros en Tailandia. En dicho estudio se encontró que mientras la autoeficacia general se relacionaba significativamente con la satisfacción vital, sólo una de las tres áreas de apoyo social percibido se relacionaba significativamente con la satisfacción vital. También este resultado puede relacionarse con lo encontrado en el estudio de Avendaño y Barra (2008) en adolescentes portadores de enfermedades crónicas, donde se obtuvo que la calidad de vida presentaba una alta relación positiva con la autoeficacia y una asociación positiva mucho menor con el apoyo social percibido. Y además puede relacionarse indirectamente con lo informado por Barra (2012) en un estudio en universitarios chilenos, donde se encontró que la autoestima tenía una influencia mayor que el apoyo social percibido sobre el nivel de bienestar psicológico.

El hecho de que la autoeficacia esté más relacionada con la satisfacción vital de los adolescentes que el apoyo social percibido podría tener al menos dos explicaciones complementarias. Por una parte, dada la importancia de la construcción de la identidad como tarea principal en esta etapa, aspectos propios de la autoeficacia como la confianza en sí mismo y la autonomía se constituirían en pilares fundamentales de la mayor o menor satisfacción con la vida de los adolescentes (Giménez et al., 2010). Y por otra parte, la menor relación del apoyo social percibido con la satisfacción con la vida en los adolescentes podría explicarse porque en la adolescencia las estructuras de apoyo social experimentarían importantes cambios, desde la familia hacia los pares y amigos, lo cual puede producir inestabilidad en las percepciones de apoyo (Avendaño y Barra, 2008; Barra et al., 2006). Según esto, el apoyo social puede ser un factor más claramente relacionado con el bienestar en los adultos que en los adolescentes, algo que debe dilucidar la investigación futura. 
Al comparar las relaciones entre las variables estudiadas en ambos sexos, se observa que la satisfacción vital presenta una relación algo mayor con la autoeficacia en los hombres adolecentes y una relación algo mayor con el apoyo de tipo instrumental en las mujeres adolescentes. Ello podría deberse a las diferencias en la socialización y los diferentes papeles de las mujeres y de los hombres dentro de la sociedad, aspectos especialmente acentuados durante la etapa adolescente. Así, mientras que en los hombres la satisfacción vital se relacionaría más con los rasgos de autoconfianza, autonomía e independencia que se enfatizan más en su socialización, a las mujeres se las socializa para ser más dependientes y buscar la ayuda de otros, por lo cual se esperaría que ellas perciban y también valoren en mayor grado que los hombres el apoyo instrumental disponible y ello se relacione con una mayor satisfacción vital.

La principal limitación de este estudio es su carácter descriptivo-correlacional y transversal, lo cual no permite establecer relaciones causales entre las variables estudiadas, ni tampoco permite determinar la influencia de otras variables que pudieran estar involucradas. Ante lo cual se sugiere para futuras investigaciones realizar estudios prospectivos que permitan determinar el valor predictivo de las variables estudiadas e incluir nuevas variables independientes que contribuyan a aumentar el conocimiento respecto a la satisfacción vital de los adolescentes.

Se espera que los resultados de este estudio aporten conocimientos que contribuyan a privilegiar un enfoque de desarrollo positivo de la adolescencia, que oriente el diseño e implementación de programas institucionales y políticas publicas que permitan el abordaje integral del adolescente en todas las áreas de funcionamiento, algo especialmente relevante en los establecimientos educacionales. De esa manera se podrían promover aquellos factores orientados a aumentar el bienestar de los adolescentes, contribuyendo de esta manera a su desarrollo integral y facilitando una adecuada transición a la vida adulta.

\section{Referencias}

Álvarez, E., y Barra, E. (2010). Autoeficacia, estrés percibido y adherencia terapéutica en pacientes hemodializados, Ciencia y Enfermería, 17, 63-72.

Antaramian, S., y Huebner, S. (2009). Stability of adolescents' multidimensional life satisfaction reports. Journal of Psychoeducational Assessment, 27, 421-425

Avendaño, M., y Barra, E. (2008). Autoeficacia, apoyo social y calidad de vida en adolescentes con enfermedades crónicas. Terapia Psicológica, $26,165-172$.

Barra, E. (2004). Apoyo social, estrés y salud. Psicología y Salud, 14, 237-243.
Barra, E. (2012). Influencia de la autoestima y del apoyo social percibido sobre el bienestar psicológico de estudiantes universitarios chilenos. Diversitas, 8, 29-38.

Barra, E., Cerna, R., Kramm, D., y Veliz, V. (2006). Problemas de salud, estrés, afrontamiento, depresión y apoyo social en adolescentes. Terapia Psicológica, 24, 55- 61.

Cid, P., Orellana, A., y Barriga, O. (2010). Validación de la Escala de Autoeficacia General en Chile. Revista Médica de Chile, 138, 551-557.

Diener, E., Scollon, C. N., y Lucas, R. E. (2009). The evolving concept of subjective well-being. En E. Diener (Ed.). Assesing well-being. The collected works of Ed Diener (pp. 67-100). Nueva York: Springer.

Galindez, E., y Casas, F. (2011). Adaptación y validación de la MSLSS de satisfacción vital multidimensional con una muestra de adolescentes. Revista de Psicología Social, 26, 309-323.

Giménez, M., Vázquez, C., y Hervás, G. (2010). El análisis de las fortalezas psicológicas en la adolescencia: Más allá de los modelos de vulnerabilidad. Psychology, Society y Education, 2, 97-116.

Gracia, E., Herrero, J., y Musitu, G. (2002). Evaluación de recursos y estresores psicosociales en la comunidad. Madrid: Editorial Síntesis.

Huebner, E. S. (2001). Manual for the multidimensional students' life satisfaction scale. Recuperado desde desde http://www.psych.sc.edu/ pdfdocs/huebslssmanual.doc

Karademas, E. (2006). Self-efficacy, social support and well-being: The mediating role of optimism. Personality and Individual Differences, 40, 1281-1290.

Luna, A., Laca, F., y Mejía, J.C. (2011). Bienestar subjetivo y satisfacción con la vida de familia en adolescentes mexicanos de Bachillerato. Psicología Iberoamericana, 19, 17-26.

Marrero, R., y Carballeira, M. (2010). El papel del optimismo y del apoyo social en el bienestar subjetivo. Salud Mental, 33, 39-46.

Mella, R., González, L., D’Appolonio, J., Maldonado, I., Fuenzalida, A., y Díaz, A. (2004). Factores asociados al bienestar subjetivo en el adulto mayor. Psykhe, 13, 79-89.

Méndez, P., y Barra, E. (2008). Apoyo social percibido en adolescentes infractores de ley y no infractores. Psykhe, 17, 59-64.

Moreno, M., y Hidalgo, M. (2010). Medición y predicción de la satisfacción con la vida en inmigrantes empleados en España. Diferencias según su situación administrativa. Anales de Psicología, 27, 179-185.

Oliva, A., Ríos, M., Antolín, L., Parra, A., Hernando, A., y Pertegal, M. (2010). Más allá del déficit: Construyendo un modelo de desarrollo positivo adolescente. Infancia y Aprendizaje, 33, 1-12.

Olivari, C., y Barra, E. (2005). Influencia de la autoeficacia y la autoestima en la conducta de fumar en adolescentes. Terapia Psicológica, 23, 5-11.

Orcasita, L., y Uribe, A. (2010). La importancia del apoyo social en el bienestar de los adolescentes. Psychologia: Avances de la Disciplina, 4, 69-82.

O'Sullivan, G. (2011). The relationship between hope, eustress, selfefficacy, and life satisfaction among undergraduates. Social Indicators Research, 101, 155-172.

Proctor, C. L., Linley, P. A., y Maltby, J. (2009). Youth life satisfaction: A review of the literature. Journal of Happiness Studies, 10, 583-630.

Reina, M., Oliva, A., y Parra, A. (2010). Percepciones de autoevaluación: Autoestima, autoeficacia y satisfacción vital en la adolescencia. Psychology, Society y Education, 2, 47-59.

Suldo, S., y Huebner, E. S. (2006). Is extremely high life satisfaction during adolescence advantageous? Social Indicators Research, 78, 179-203.

Vega, O., y González, D (2009). Apoyo social: Elemento clave en el afrontamiento de la enfermedad crónica. Enfermería Global, 16, 1-11.

Vera-Villarroel, P., Urzúa, A., Pavez, P., Celis-Atenas, K., y Silva, J. (2012). Evaluation of subjective well-being: Analysis of the Satisfaction with Life Scale in Chilean population. Universitas Psychologica, 11, 719-727.

Yusoff, Y. (2012). Self-efficacy, perceived social support, and psychological adjustment in international undergraduate students in a public higher education institution in Malaysia. Journal of Studies in International Education, 16, 353-371 